\title{
Single flux quantum circuits with damping based on dissipative transmission lines
}

\author{
E. M. Tolkacheva * D. V. Balashov, M. I. Khabipov, and A. B. Zorin \\ Physikalisch-Technische Bundesanstalt, Bundesallee 100, 38116 Braunschweig, Germany
}

(Dated: April 2, 2008)

\begin{abstract}
We propose and demonstrate the functioning of a special Rapid Single Flux Quantum (RSFQ) circuit with frequency-dependent damping. This damping is achieved by shunting individual Josephson junctions by pieces of open-ended $R C$ transmission lines. Our circuit includes a toggle flip-flop cell, Josephson transmission lines transferring single flux quantum pulses to and from this cell, as well as DC/SFQ and SFQ/DC converters. Due to the desired frequency-dispersion in the $R C$ line shunts which ensures sufficiently weak damping at low frequencies, such circuits are well-suited for integrating with the flux/phase Josephson qubit and enable its efficient control.
\end{abstract}

\section{INTRODUCTION}

The Josephson circuits operating on Single Flux Quanta (SFQ), the so-called Rapid Single Flux Quantum (RSFQ) circuits [1] of special design, are intensively investigated as they are the most promising on-chip system enabling the control and readout of the superconducting qubits 2, 3]. This choice is primarily based on the well developed fabrication technology of these circuits, allowing them to be implemented in the same process together with the Josephson qubits. Moreover, the capability of the RSFQ circuits to function at the same low temperature as the qubits is another advantage of this approach. Among these RSFQ circuits are the balanced comparators [4, 5, 6], SQUIDs 7], the ballistic Josephson Transmission Lines (JTL) [, 9] enabling quantum readout of the qubit, and the RSFQ circuits generating signals controlling the qubit state [10, 11].

One of the most important issues in integrating the RSFQ circuits with a qubit is their strong back action on the qubit. On the one hand, the operating principle of the RSFQ circuits assumes the availability of significant damping which is usually ensured by the low-ohmic resistive shunting $R$ of the Josephson Superconductor Insulator - Superconductor (SIS) tunnel junctions (see the electric diagram in Fig.1(a), where the cross denotes the junction itself with a presumably very high quasiparticle resistance $R_{\mathrm{qp}} \gg R$ ). On the other hand, such resistive shunts generate significant current noise with the white spectrum even in the quiescent (zero-voltage) state of the Josephson junctions. This noise is admixed to the bias and output control signals and, therefore, may dramatically decohere the qubit [12, 13]. A possible solution to this problem is the application of frequency-dependent elements which ensure sufficient damping at plasma resonance frequency $\nu_{p}=\omega_{p} / 2 \pi \sim 40 \mathrm{GHz}$ of the junctions of the RSFQ circuit, but have a relatively low damping and, therefore, a low noise at frequencies below the characteristic frequency of the qubit $\nu_{01}=\left(E_{1}-E_{0}\right) / h \lesssim 10 \mathrm{GHz}$. As we had shown earlier [14, 15], to realize such damping, one can use non-linear shunts on the basis of Superconductor - Insulator - Normal metal (SIN) tunnel junc-

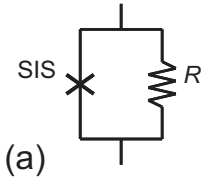

(b)

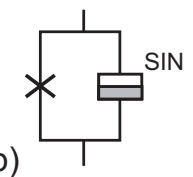

(c)

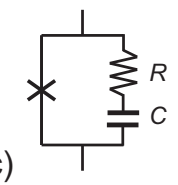

(d)
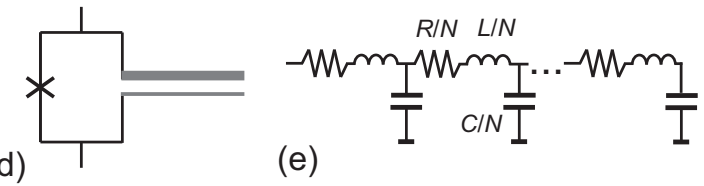

(e)

FIG. 1: Different ways of realizing the damping in Josephson SIS junctions: (a) resistive shunting, (b) shunting by an SIN tunnel element, (c) shunting by an $R C$ circuit, and (d) shunting by an open-ended dissipative transmission line. (e) The equivalent electric diagram of such transmission line modeled by the ladder circuit consisting of sufficiently large number $N$ of elementary $R L C$ sections.

tions (see Fig.1(b)). Although these shunts may ensure both a remarkably large high-frequency damping and a significant suppression of the low-frequency noise due to a presumably small sub-gap leakage current in the SIN junctions [16], the fabrication technology of these hybrid SIS+SIN circuits is rather difficult.

Recently, a simpler realization of RSFQ networks with a frequency-dependent damping based on shunting the junctions by serial $R C$ circuits (see Fig.1(c)) has been proposed in Ref. 17] and verified in Ref. [18]. This linear circuit consisting of thin-film resistor and plate on-chip capacitor is relatively simple for fabrication. Application of such shunting elements was beneficial in the low-noise dc SQUIDs used in magnetometry [19] and in the readout SQUIDs for the Josephson qubit [20].

In this paper, we propose to damp an RSFQ circuit by shunting its junctions by pieces of dissipative transmission lines (see Fig.1(d)-(e)). We report the results of numerical simulations and of the measurements of a $\mathrm{Nb}$ RSFQ circuit provided with such damping. Our experimental circuit includes Toggle Flip-Flop (TFF), Josephson transmission lines (JTL) and DC/SFQ and SFQ/DC converters. We will show that a microstrip line shunts 
made of a resistive film which is normally used for the fabrication of shunting resistors can ensure a stable operation of the circuit with a desirable frequency-dependent damping and give advantages in the suppression of noise at typical qubit frequencies.

\section{MODEL AND SIMULATIONS}

The strength of the damping in Josephson circuits is determined by power losses at the frequency of plasma resonance $\omega_{p}=\left(L_{J} C_{J}\right)^{-1 / 2}$, where $L_{J}=\Phi_{0} /\left(2 \pi I_{c}\right)$ is the Josephson inductance, $\Phi_{0}$ the flux quantum, $I_{c}$ the critical current and $C_{J}$ the junction capacitance. When the junction is shunted by an ohmic resistor (Fig.1(a)), the damping is characterized by the dimensionless McCumber-Stewart parameter [21, 22],

$$
\beta_{c}=\left(R / \omega_{p} L_{J}\right)^{2}=\left(2 \pi / \Phi_{0}\right) I_{c} R^{2} C_{J} .
$$

To ensure a normal operation of an RSFQ circuit, its value must be sufficiently small, i.e. $\beta_{c} \lesssim 2$ (overdamped regime). In the case of frequency-dependent shunting (shown in Fig.1(b)-(e)), the resulting damping depends on the frequency dispersion of the shunting element and on the parameters of the junction, i.e. $L_{J}$ and $C_{J}$. Generally, the complex admittance of the shunt, $Y(\omega)=Y^{\prime}(\omega)+j Y^{\prime \prime}(\omega)$, adds not only the losses $Y^{\prime}(\omega)$, but causes also a shift of the plasma resonance frequency $\omega_{p} \rightarrow \tilde{\omega}_{p}$ (see, e.g., Ref. [15]). This effective plasma frequency $\tilde{\omega}_{p}$ is the root of the following equation:

$$
\omega\left[C_{J}+C^{*}(\omega)\right]-1 /\left(\omega L_{J}\right)=0,
$$

where $C^{*}(\omega)=Y^{\prime \prime}(\omega) / \omega$. The effective dimensionless damping parameter is, therefore, equal to

$$
\tilde{\beta}_{c}=\left(R^{*} / \tilde{\omega}_{p} L_{J}\right)^{2}=\left[\tilde{\omega}_{p} L_{J} Y^{\prime}\left(\tilde{\omega}_{p}\right)\right]^{-2},
$$

and this value may reduce to the desirable level of about 1 when the effective shunting resistance $R^{*}(\omega) \equiv 1 / Y^{\prime}(\omega)$ approaches the high-frequency values $\omega \sim \tilde{\omega}_{p}$ which lie above the roll-off frequency $\sim(R C)^{-1}$.

The open-ended transmission lines $\left(Y_{\text {load }}=0\right)$ with the total resistance $R$, the total capacitance $C$ and the total inductance $L$, which we apply as Josephson junction shunts, have the following parameters per unit length: $r=R / \ell, c=C / \ell$ and $l=L / \ell$. The length $\ell$ is smaller than the wavelength $\lambda_{p}$ corresponding to the characteristic frequencies $\left(\sim \tilde{\omega}_{p}\right)$ of the problem. We exclude from our analysis the leakage of dielectric and will normally omit the effect of the line inductance $L$ which, as we show below, manifests itself at frequencies well above the operation frequency of the RSFQ circuit. Applying the transmission-line theory, the admittance seen by the junction is expressed as

$$
Y(\omega)=(j \omega C / R)^{1 / 2} \tanh \left[(j \omega R C)^{1 / 2}\right] .
$$

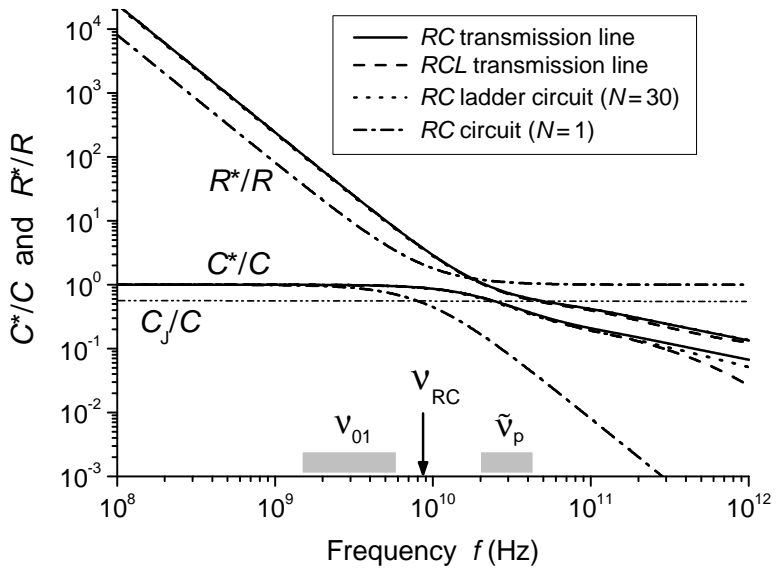

FIG. 2: Frequency dependencies of the normalized effective capacitance $C^{*}$ and the normalized effective resistance $R^{*}$ in different types of shunting circuits, calculated for $R=10 \Omega$ and $C=1.8 \mathrm{pF}$, yielding the transition frequency $\nu_{R C} \equiv$ $(2 \pi R C)^{-1} \approx 8.8 \mathrm{GHz}$. The inductance of the distributed $R C L$ transmission line (dashed lines) is $L=1.9 \mathrm{pH}$. For comparison, dashed-dotted lines show the behavior of $C^{*}$ and $R^{*}$ in a simple $R C$ circuit with similar $R$ and $C$. The thin dasheddotted-dotted horizontal line shows the value of the junction capacitance $C_{J}$. The grey color bars show the typical frequency ranges of a qubit $\nu_{01}$ and of an RSFQ circuit $\tilde{\nu}_{p}$.

The plots of the normalized effective shunting resistance $R^{*}(\omega) / R$ and the effective capacitance $C^{*}(\omega) / C$ calculated using Eq. (44) are presented in Fig. (2) by solid lines.

The parameters of the frequency-dependent shunting circuits, whose characteristics are shown in Fig.2, were chosen such that the characteristic frequency of the qubit $\nu_{01}$ (of several $\mathrm{GHz}$ ) lay notably below the effective plasma frequency $\tilde{\nu}_{p}=\tilde{\omega}_{p} / 2 \pi \approx 20-40 \mathrm{GHz}$. This value of $\tilde{\nu}_{p}$ was estimated from the designed value of the critical current $I_{c}=12 \div 24 \mu \mathrm{A}$, the self-capacitance of the junctions $C_{J}=0.5 \div 1 \mathrm{pF}$ (corresponding to the junction area $A=12 \div 24 \mu \mathrm{m}^{2}$ and the critical current density $j_{c}=100 \mathrm{~A} / \mathrm{cm}^{2}$ in the $\mathrm{Nb}$ multilayer process of $\mathrm{PTB}$ [23]). The transmission line was realized in the resistive composite layer consisting of a sandwich $\mathrm{Cr} / \mathrm{Pt} / \mathrm{Cr}$. The nominal sheet resistance of this film was $R_{\square}=2 \Omega$.

The numerical evaluation of $R, C$ and $L$ in the transmission line with the lateral dimensions $30 \mu \mathrm{m}$ by $150 \mu \mathrm{m}$ yielded an optimum resistance value of $10 \Omega$ and an optimum capacitance of $1.8 \mathrm{pF}$. Evaluation of the transmission line inductance applying the Maxwell software package 24] yielded the sufficiently small value of $L=1.9 \mathrm{pH}$. The effect of this inductance, as can be seen from Fig.2 (the corresponding curves are shown by dashed lines), is negligibly small in the interesting range of frequencies (up to 200-300 GHz, exceeding the plasma frequency $\tilde{\nu}_{p}$ by one order of magnitude). Therefore, in our circuit simulations, we assumed a zero inductance $L=0$ 
(a)

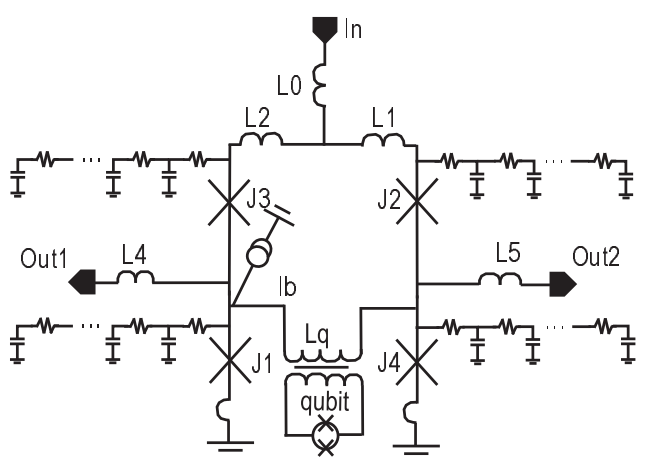

(b)

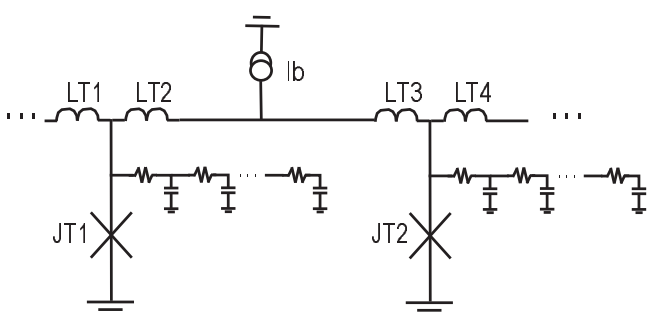

FIG. 3: Schematics of the basic RSFQ circuits with $R C$ transmission line shunts: (a) TFF, intended for the control of a Josephson qubit in loop configuration, with a possible inductive coupling to it (shown in the middle) and (b) one section of JTL.

which resulted in a pure diffusive behavior of the transmission lines. A ladder network model shown in Fig.1(e) with the number of sections $N=30$ gave very good approximation of the distributed line (dotted lines in Fig.22). This model made it possible to easily include these lumped-element networks into the software package PSCAN [25] and to simulate the behavior of our circuits with frequency-dependent damping. For the sake of a further simplification of the modeling, the circuit with $N=10$ sections (having very similar characteristics in the interesting range of frequencies, not shown in Fig.(2) was also extensively applied in the circuit optimization.

We have modeled the behavior of the basic RSFQ circuits including TFF (shown in Fig.3(a)) and JTL (see Fig. (3)(b)) with $R C$ transmission line shunts. Such TFF circuit has been developed for the control of the Josephson qubit in SQUID configuration so its quantizing inductance $L_{q}$ is magnetically coupled to the qubit loop. As has recently been demonstrated in Ref. [11], the output rectangular magnetic flux pulses of TFF, triggered by the incoming SFQ pulses at the port "In", can effectively change the shape of the energy potential of the double-SQUID flux qubit and, hence, form the basis for the quantum manipulation. For this type of Josephson qubit, a more than 300-fold increase in the effective shunting resistance can be achieved at its typical frequency $\nu_{01} \approx 1 \mathrm{GHz}$. The absolute value of $R^{*}$ in our design is $3 \mathrm{k} \Omega$ at this frequency. Note that according to

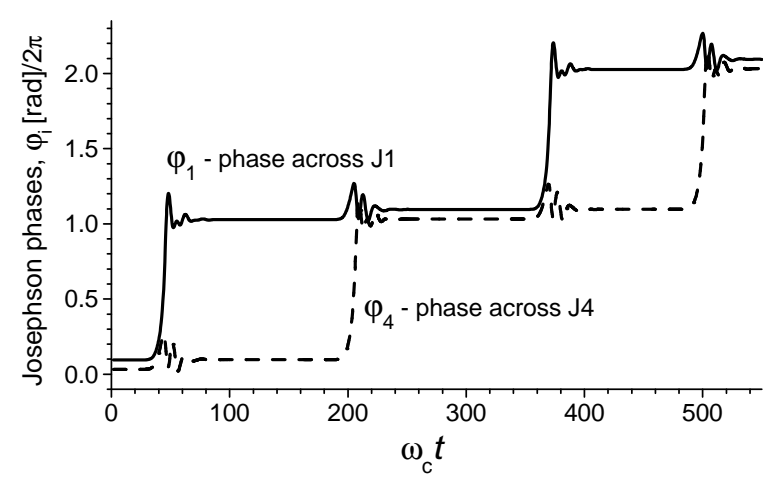

FIG. 4: Josephson phases across the junctions J1 and J2 of TFF with $R C$ line shunts (Fig. [3(a)), obtained by numerical simulations. The characteristic circular frequency used for normalizing the time scale is $\omega_{c}=(20 \mathrm{ps})^{-1}$.

estimations made by Chiarello [26] taking into account the effect of attenuation of noise due to sufficiently weak coupling, the resistance $R^{*}$ kept at sufficiently low temperature of $20 \mathrm{mK}$ must not be lower than $100 \Omega$. In this case, decoherence of the qubit is still tolerable. Therefore, the designed $R C$ transmission line shunts are very well-suited for controlling this qubit.

The result of the simulations for the set of parameters typical for our technology is shown in Fig. 4 as time dependencies of the Josephson phases reacting on the input SFQ pulses. The step-like behavior of the phases $\varphi_{1}$ and $\varphi_{4}$ on the Josephson junctions $J 1$ and $J 4$, respectively, is due to $2 \pi$ phase leaps corresponding to the switching of the TFF between two states. An incoming SFQ pulse (not shown here) induces $2 \pi$ phase leaps across the junctions $J 1$ and $J 2$ (see Fig. (3); the next SFQ pulse causes $2 \pi$ leaps across junctions $J 3$ and $J 4$. This yields the sequence of current pulses in the storing inductance $L_{q}$, and therefore the pulses of the output magnetic flux. These pulses have required rectangular shape (see, for comparison, similar plots in Fig. 7(b) of Ref. [15], obtained for SIN-junction-shunted TFF). The parameter margins found in the optimization of the circuit are in the safe range typical of RSFQ circuits [27]; the most critical parameter is the critical current density $j_{c}$, having margins of $\pm 28 \%$.

\section{EXPERIMENT}

The circuit designed for the experimental testing of the functionality of the $R C$ transmission line shunts is the TFF cell which is similar to that shown in Fig. 3, but its input and two outputs were attached to JTLs with $R C$ line shunts serving as buffer stages. In the block diagram in Fig. 5 (a), this part of the circuit is marked by grey color and placed inside the dashed-line box. Due to the 
frequency-dependent damping, such a JTL can transmit SFQ pulse signals and efficiently attenuate noise originating from the external part of the circuit. Each JTL circuit includes two sections which ensure an attenuation of the noise coming from the conventional resistive shunts of an external circuit by more than $40 \mathrm{~dB}$. Such a large attenuation was possible due to the relatively low currents biasing the sections of the JTL, which resulted in rather small values of the Josephson inductances and, therefore, a division of signals with large ratio. The "external" part of the circuit includes four-section JTLs with resistive shunts as well as DC/SFQ and SFQ/DC converters. The DC/SFQ converter is used for generating the sequence of the SFQ pulses driving the TFF, while two SFQ/DC converters enable visualization of the TFF switching. The SFQ pulses transmitted via two output channels of the TFF are therefore converted into rectangular voltage pulses. The edges of these rectangular pulses are time-referenced to the input SFQ pulses and the repetition frequency is four times lower than the frequency of the input pulses. Moreover, the two output signals have a mutual phase delay. This behavior must originate from the pulse rate division by two sequentially connected TFFs. The first one is the core TFF with $R C$ transmission-line-shunted junctions, and the second one in each line is the TFF of conventional design with integrated SFQ pulse detector with resistively shunted junctions. The mutual shift of the output signals $V_{\text {out1 }}$ and $V_{\text {out2 }}$ should correspond to exactly one period of the input signal $I_{\text {in }}$.

The fabrication process included the deposition of an Nb film (170 nm thick) which was patterned for structuring a ground plane of the circuits. The electrical insulation of the ground plane was provided by a wet anodization of the $\mathrm{Nb}$ film, followed by the sputtering of a dielectric $\mathrm{SiO}_{2}$ layer (80 nm thick) on top of it. To form the bias and shunt resistors, we deposit a $\mathrm{Cr} / \mathrm{Pt} / \mathrm{Cr}(10$ $\mathrm{nm} / 43 \mathrm{~nm} / 10 \mathrm{~nm}$ ) sandwich patterned by an Ar-milling etch process. Thereafter, a second $\mathrm{SiO}_{2}$ ground plane insulation layer (140 $\mathrm{nm}$ thick) was sputtered. In order to connect the groundplane and the resistors to other metal layers, holes were etched in this dielectric layer. The $\mathrm{Nb} / \mathrm{Al}-\mathrm{Al}_{x} \mathrm{O}_{y} / \mathrm{Nb}(170 \mathrm{~nm} / 10 \mathrm{~nm} / 80 \mathrm{~nm})$ trilayer was deposited in an UHV system with a base pressure lower than $10^{-6} \mathrm{~Pa}$. The oxidation process was performed in a load-lock with a base pressure lower than $10^{-5} \mathrm{~Pa}$. The tunnel areas of the Josephson junctions were formed by etching the $\mathrm{Nb}$ counter electrode of the trilayer. Then, the wet anodization process was applied again, followed by the patterning of the $\mathrm{Nb}$ base electrode. $\mathrm{An} \mathrm{SiO}_{2}(300$ $\mathrm{nm}$ ) layer was sputtered onto the sample for insolating the edges of the base electrode and strengthening the anodic oxide. The process was completed by sputtering an $\mathrm{Nb}$ (400 nm) wiring layer. The optical microscope image of a fragment of the finished sample is shown in Fig.5(b). The $R C$ transmission line shunts of rectangular shape are seen in this image.

The measurements of the samples were performed at a liquid helium temperature of $T=4.2 \mathrm{~K}$. At first, we tested the stand-alone Josephson junctions with $R C$ transmission line shunts which were fabricated on the same chip for the purpose of measuring their autonomous $I-V$ characteristics. Figure6(a) shows a typical $I-V$ curve, exhibiting rather strong hysteresis. The critical current $I_{c}$ with a nominal value of $16.6 \mu \mathrm{A}$ is notably
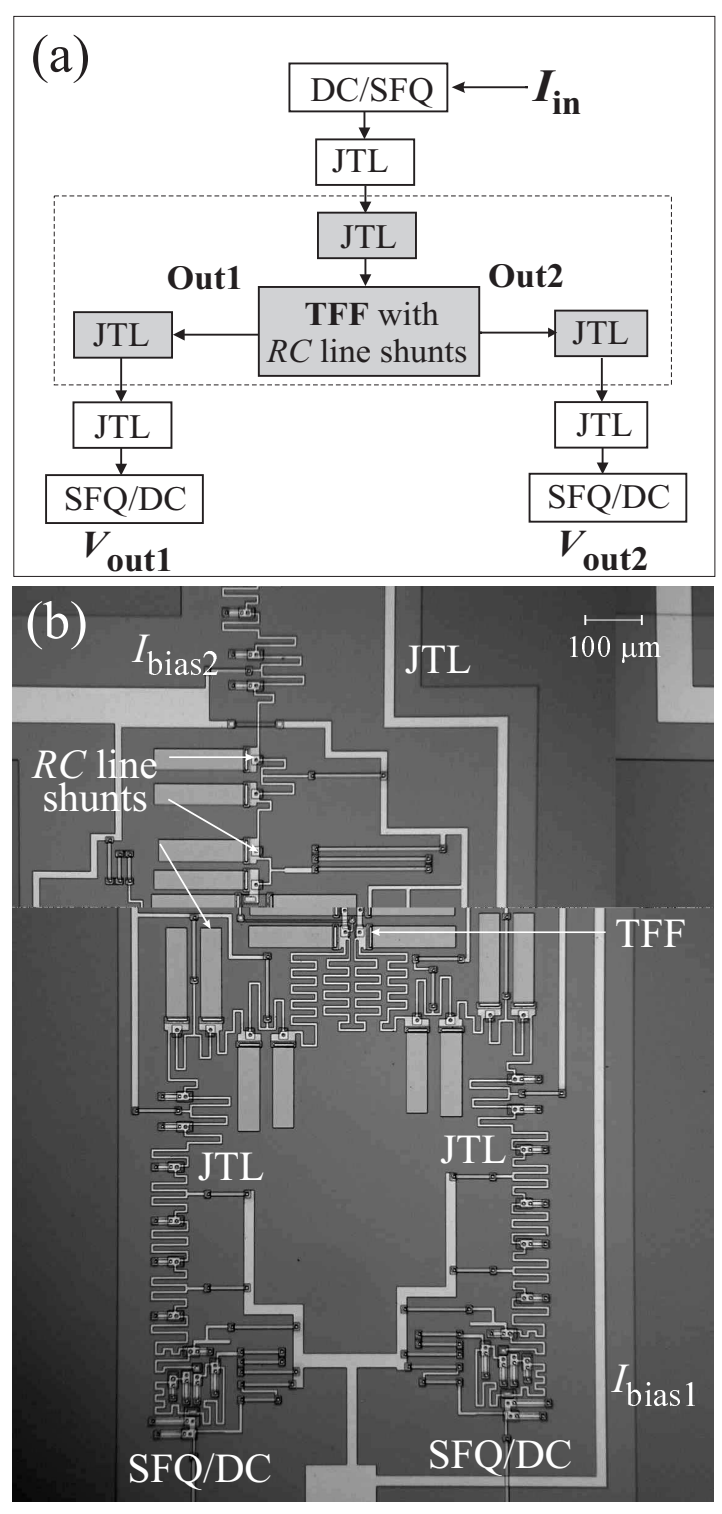

FIG. 5: (a) Block diagram of the circuit with $R C$ line shunts (elements inside the dashed-line box) and resistive shunts (outside this box) including TFF, JTL, DC/SFQ and SFQ/DC converters. (b) Image of the circuit fabricated at $\mathrm{PTB}$ in Nb technology with $j_{c}=100 \mathrm{~A} / \mathrm{cm}^{2}$. Critical currents of the TFF junctions are $I_{c}=17 \mu \mathrm{A}$ each, the quantizing inductance $L_{q}=132 \mathrm{pH}$. 
(a)

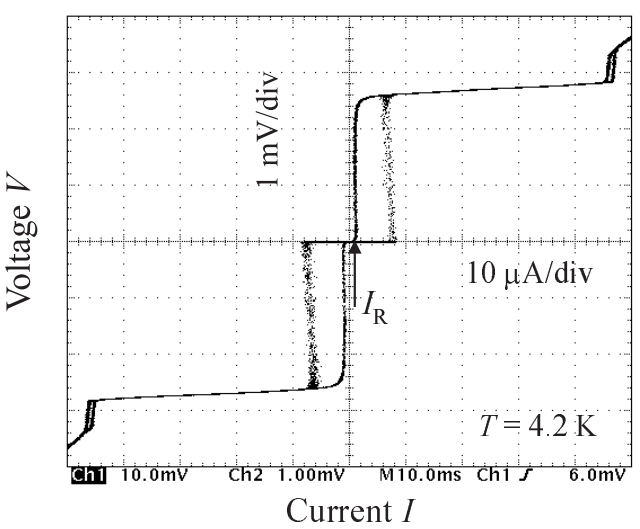

(b)

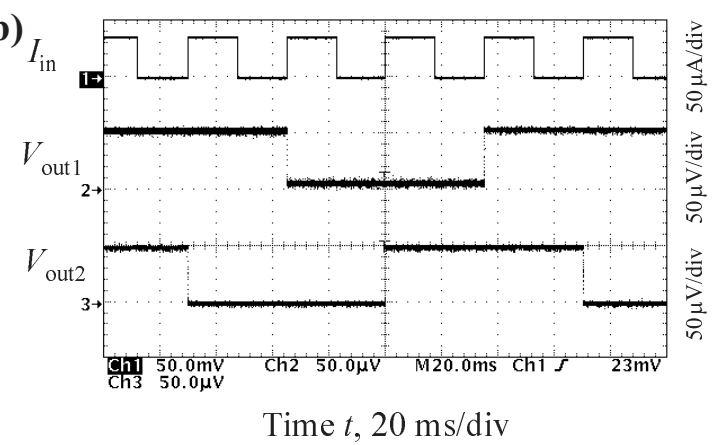

FIG. 6: (a) Autonomous $I-V$ curve of the $R C$-line-shunted Josephson junction. (b) Time traces of the signals in the TFF circuit with $R C$-line-shunted junctions. The frequency of the output signals $V_{\text {out1 }}$ and $V_{\text {out2 }}$ is four times lower compared with the frequency of the input signal $I_{\text {in. }}$. The observed division of the frequency of the input pulses indicates that the TFF operates correctly.

suppressed due to the noise in the measuring lines, so the switching into the resistive state occurs at $I \approx 8 \mu \mathrm{A}$. The value of the return current $I_{R}$, determined by both the junction capacitance $C_{J}$ and the shunting admittance $Y(\omega)$, is about $1 \mu \mathrm{A}$. Such a value of $I_{R}$ corresponds in the resistively-shunted model of the junction [21, 22] to the value of $\beta_{c} \approx\left(4 I_{c} / \pi I_{R}\right)^{2} \approx 200$. In the quiescent (zerovoltage) state of the circuit, the zero-bias quasiparticle resistance $R_{\mathrm{qp}}$ has to be taken into account for evaluating the low-frequency noise. The values of this resistance are estimated to be higher than $2 \mathrm{k} \Omega$ for the stand-alone junctions and $1 \mathrm{k} \Omega$ for the junctions used in the TFF circuit. Note that the equivalent value of $\beta_{c}$, derived for an unshunted junction with $R_{\mathrm{qp}}=1 \mathrm{k} \Omega$, is $5 \times 10^{4}$, while the effective value $\tilde{\beta}_{c}$ designed for the junction shunted by the $R C$ transmission line is about 1 . This value ensures a sufficient damping in the dynamic regime.

Figure6(b) demonstrates the signal time traces in the circuit shown in Fig. 5 . The output signals $V_{\text {out1 }}$ and $V_{\text {out2 }}$ shifted with half a period and with frequency four times lower than the frequency of the input signal $I_{\text {in }}$ confirm that the circuits operate correctly. The range of its operation was found to be sufficiently wide. The margins for the bias currents were $\pm 21 \%$ for the $R C$-lineshunted TFF circuit $\left(I_{\text {bias2 } 2}\right.$, see Fig. $5($ (b) $)$ and $\pm 30 \%$ for the bias of the DC/SFQ and SFQ/DC converters based on resistively shunted junctions $\left(I_{\text {bias1 }}\right)$.

\section{DISCUSSION}

One of the complex issues in designing an integrated "RSFQ + qubit" circuit operating at millikelvin temperature is the overheating of the shunting resistors due to the dissipated power $P \approx f I_{c} \Phi_{0}$, where $f$ is the working (clock) frequency [28]. Because of the rather small volume $\Lambda_{R} \approx 10 \mu \mathrm{m}^{3}$ of conventional thin film resistors (they must have a relatively small stray capacitance) the effective electron temperature $T_{e} \approx\left(P / \Sigma \Lambda_{R}\right)^{1 / 5}$ (here $\Sigma \sim 1 \mathrm{nW} \mu \mathrm{m}^{-3} \mathrm{~K}^{-5}$ is the material constant) may significantly exceed the bath temperature even at low $f[5]$. The efficient method proposed in Ref. [5] of reducing $T_{e}$ was based on the application of bulky metallic reservoirs $\left(\Lambda_{f} \approx 4 \times 10^{5} \mu \mathrm{m}^{3}\right)$, so-called cooling fins, attached to the small resistors. The volume of the $R C$ line shunts designed in our paper is $\Lambda_{R C} \approx 284 \mu \mathrm{m}^{3} \approx 30 \Lambda_{R}$, and this increased volume should result in an approximately twofold $\left(30^{\frac{1}{5}} \approx 2\right)$ reduction in their electron temperature $T_{e}$ without attaching them to cooling fins. Moreover, the large size of the $R C$ line shunts makes it, in principle, possible to attach cooling fins of larger volume. These cooling fins may have a more efficient thermal contact to the large "hot" area of the $R C$ line shunt than in the case of relatively small resistive shunts or the $R C$ shunts [18].

Another advantage of the $R C$ transmission line shunts over the $R C$ shunts consists in inherently diffusive transmission characteristics of these lines and, therefore, the absence of parasitic electromagnetic resonances due to standing waves in these structures. These resonances may present a problem in Nb-based on-chip capacitors having very low losses. (The $R C$ shunts with such capacitors were realized in VTT technology with a critical current density $j_{c}=30 \mathrm{~A} / \mathrm{cm}^{2}$ [18].) Therefore, the lateral dimensions of the capacitors $(100 \mu \mathrm{m}$ by $160 \mu \mathrm{m}$ with $140 \mathrm{~nm}$ thick $\mathrm{Nb}_{2} \mathrm{O}_{5}$ dielectric yielding $C=35 \mathrm{pF}$ [18]) should not exceed $\lambda_{p} / 8(\approx 170 \mu \mathrm{m})$, where $\lambda_{p}$ is the electromagnetic wavelength in the dielectric at the plasma frequency.

Comparison of both the levels of the low-frequency noise and the efficiencies of the high-frequency damping in the $R C$ transmission line shunt and $R C$ shunt yields the following results. For similar values of the parameters $R$ and $C$ in both models, the effective conductance $Y^{\prime}=1 / R^{*}$ at $\omega \ll(R C)^{-1}$ is equal to $\omega^{2} C^{2} R$ and $\omega^{2} C^{2} R / 3$ (given by Eq. (4) in the low-frequency limit), respectively. This difference is also to be seen in the plot in Fig.2, For the equal effective temperatures of these 
shunts, this yields a three-times lower spectral density of the current noise $\left(=4 k_{B} T_{e} Y^{\prime}\right)$. There is also a difference in the behavior at high frequencies, $\omega \gg(R C)^{-1}$. In this frequency range, the effective capacitance of the system "junction + shunt" is mostly determined by the capacitance of the junction $C_{J}$ (compare the behavior of $C^{*}$ shown by the solid and dashed-dotted lines with the typical level of $C_{J}$ shown by thin dashed-dotted-dotted line in Fig.(2). Therefore, the strength of the damping is determined by the shunt losses only. The $R C$ transmission line model yields, therefore, the appreciably larger damping, $R^{*}=(2 R / \omega C)^{1 / 2} \ll R$ (high-frequency limit in Eq. (44) than the plain damping $R$ in the $R C$ shunt model. The enhanced high-frequency damping should stabilize the operation of the RSFQ circuits with the $R C$ transmission line shunts.

In conclusion, we developed an efficient approach to the problem of the frequency-dependent damping of RSFQ circuits to be used for integration with Josephson qubits. The proposed junction shunts based on $R C$ transmission lines have remarkable electric characteristics and, as we have demonstrated, can be easily implemented in the framework of the available $\mathrm{Nb}$ technology [23].

\section{ACKNOWLEDGEMENTS}

We are grateful to Boyko Dimov for assistance in simulations and Friedrich-Immanuel Buchholz, Gabriella Castellano and Fabio Chiarello for helpful discussions. This work was partially supported by the EU through the RSFQubit and EuroSQIP projects.

* Present address: D-wave Systems Inc., 100-4401 Still Creek Drive, Burnaby, B.C. V5C 6G9, Canada

[1] K. K. Likharev and V. K. Semenov, IEEE Trans. Appl. Supercond. 1, 3 (1991).

[2] V. K. Semenov and D. V. Averin, IEEE Trans. Appl. Supercond. 13, 960 (2003).

[3] G. Wendin and V. S. Shumeiko, in Superconducting quantum circuits, qubits and computing, Handbook of Theoretical and Computational Nanotechnology ed. M. Rieth and W. Schommers (ASP, Los Angeles, 2006), vol. 3, 223.

[4] M. Wulf, X. Zhoi, J. L. Habif, P. Rott, M. F. Bocko, and M. J. Feldman, IEEE Trans. Appl. Supercond. 13, 974
(2003).

[5] A. M. Savin, J. P. Pekola, T. Holmqvist, J. Hassel, L. Grönberg, P. Helistö, and A. Kidiyarova-Shevchenko, Appl. Phys. Lett. 89, 133505 (2006).

[6] T. Ohki, A. Savin, J. Hassel, L. Grönberg, T. Karminskaya, and A. Kidiyarova-Shevchenko, IEEE Trans. Appl. Supercond. 17, 128 (2007).

[7] S. Li, Y. Yu, W. Qiu, S. Han, and Z. Wang, IEEE Trans. Appl. Supercond. 13, 982 (2003).

[8] D. V. Averin, K. Rabenstein, and V. K. Semenov, Phys. Rev. B 73, 094504 (2006).

[9] A. Fedorov, A. Shnirman, A. Shön, and A. KidiyarovaShevchenko, Phys. Rev. B 75, 224504 (2007).

[10] D. S. Crankshaw, J. L. Habif, X. Zhoi, T. P. Orlando, M. J. Feldman, and M. F. Bocko, IEEE Trans. Appl. Supercond. 13, 966 (2003).

[11] G. M. Castellano, F. Chiarello, R. Leoni, G. Torrioli, P. Carelli, C. Cosmelli, M. Khabipov, A. B. Zorin, and D.V. Balashov, Supercond. Sci. Technol. 2, 500 (2007).

[12] Yu. Makhlin, G. Schön, and A . Shnirman, Rev. Mod. Phys. 73, 357 (2001).

[13] C. H. van der Wal , F. K. Wilhelm, C. J. P. M. Harmans, and J. E. Mooij, Eur. Phys. J. B 31, 111 (2003).

[14] A. B. Zorin, M. I. Khabipov, D. V. Balashov, R. Dolata, F-Im. Buchholz, and J. Niemeyer, Appl. Phys. Lett. 86, 032501 (2005).

[15] A. B. Zorin, E. Tolkacheva, M. I. Khabipov, F.-Im. Buchholz, and J. Niemeyer, Phys. Rev. B 74, 014508 (2006).

[16] S. V. Lotkhov, D. V. Balashov, M. I. Khabipov, F-I. Buchholz, and A. B. Zorin, Physica C 449, 81 (2006).

[17] J. Hassel, P. Helistö, H. Seppä, J. Kunrt, L. Fritzsch, and H-G. Meyer, Appl. Phys. Lett. 89, 182514 (2006).

[18] J. Hassel, L. Grönberg, and P. Helistö, New J. Phys. 9, 157 (2007).

[19] M. Kiviranta and H. Seppä, Appl. Supercond. 6, 373 (1998).

[20] T. L. Robertson, B. L. T. Plourde, T. Hime, S. Linzen, P. A. Reichardt, F. K. Wilhelm, and J. Clarke, Phys. Rev. B 72, 024513 (2005).

[21] D. E. McCumber, J. Appl. Phys. 39, 3113 (1968).

[22] W. C. Stewart, Appl. Phys. Lett. 12, 277 (1968).

[23] R. Dolata, M. Khabipov, F-Im. Buchholz, W. Kessel, and J. Niemeyer, Proc. of the 2nd European Conf. on Appl. Supercond. 148, 1709 (1995).

[24] B. Dimov, private communication.

[25] S. V. Polonsky, V. K. Semenov, and P. N. Shevchenko, Supercond. Sci. Technol. 4, 667 (1991).

[26] F. Chiarello, Eur. Phys. J. B 55, 7 (2007).

[27] S. Intiso, I. Kataeva, E. Tolkacheva, H. Engseth, K. Platov, and A. Kidiyarova-Shevchenko, IEEE Trans. Appl. Supercond. 15, 328 (2004).

[28] A. M. Savin, J. P. Pekola, D. V. Averin, and V. K. Semenov, J. Appl. Phys. 99, 084501 (2006). 